\title{
Linking laboratory and medication data: new opportunities for pharmacoepidemiological research
}

\author{
Maarten J. ten Berg ${ }^{1,2}$, Albert Huisman², \\ Patricia M.L.A. van den Bemt ${ }^{1,3}$, Alfred F.A.M. \\ Schobben ${ }^{1,4}$, Antoine C.G. Egberts ${ }^{1,4}$ and \\ Wouter W. van Solinge ${ }^{1,2, *}$ \\ ${ }^{1}$ Department of Pharmacoepidemiology and \\ Pharmacotherapy, Utrecht Institute for \\ Pharmaceutical Sciences, Utrecht, The Netherlands \\ 2 Department of Clinical Chemistry and \\ Haematology, University Medical Centre Utrecht, \\ Utrecht, The Netherlands \\ ${ }^{3}$ Department of Clinical Pharmacy, TweeSteden \\ hospital and St. Elisabeth Hospital, Tilburg, The \\ Netherlands \\ ${ }^{4}$ Department of Clinical Pharmacy, University \\ Medical Centre Utrecht, Utrecht, The Netherlands
}

\begin{abstract}
Transfer of automated laboratory data collected during routine clinical care from the laboratory information system into a database format that enables linkage to other administrative (e.g., patient characteristics) or clinical (e.g., medication, diagnoses, procedures) data provides a valuable tool for clinical epidemiological research. It allows the investigation of biochemical characteristics of diseases, therapeutic effects and diagnostic and/or prognostic markers for disease with easy access and at relatively low cost. To this end, the Utrecht Patient Oriented Database (UPOD), an infrastructure of relational databases comprising data on patient characteristics, laboratory test results, medication orders, hospital discharge diagnoses and medical procedures for all patients treated at the University Medical Centre Utrecht since January 2004, was established. Current research within UPOD is focused on the innovative linkage of laboratory and medication data, which, for example, makes it possible to assess the quality of pharmacotherapy in clinical practice, to investigate interference between laboratory tests and drugs, to study the risk of adverse drug reactions, and to develop diagnostic and prognostic markers or algorithms for adverse drug reactions. Although recently established, we believe that UPOD broadens the opportunities for clinical pharmacoepidemiological research and can
\end{abstract}

\footnotetext{
*Corresponding author: Wouter W. van Solinge, Department of Pharmacoepidemiology and Pharmacotherapy, Utrecht Institute for Pharmaceutical Sciences, P.O. Box 80.082, 3508TB Utrecht, The Netherlands

Phone: +31-30-2507604, Fax: +31-30-2505418,

E-mail: wsolinge@umcutrecht.nl
}

contribute to patient care from a laboratory perspective.

Clin Chem Lab Med 2007;45:13-9.

Keywords: clinical chemistry; clinical epidemiology; laboratory medicine; pharmacoepidemiology; research database.

\section{Introduction}

Since the introduction of the first computers to process and capture laboratory data in the 1960s (1), enormous progress has been made in laboratory automation. Currently, the majority of biochemical laboratory tests are performed by fully automated analysers, and test results are processed and stored electronically within advanced laboratory information systems. These automated laboratory data are primarily used in patient care and for management purposes. However, transfer of data from the laboratory information system into a database format that allows questioning and linkage to administrative (e.g., patient characteristics) or other clinical data (e.g., disease and medical treatment) would provide a valuable tool for clinical epidemiological research, i.e., the application of epidemiological principles and methods to problems encountered in clinical medicine (2).

Until now, most clinical epidemiological research with laboratory data was only possible after elaborate gathering of data for a specific research question. A structurally available data linkage system that provides complete and well-defined research data that can be questioned at any time would increase the possibilities for conducting clinical epidemiological research with laboratory data. Therefore, the Utrecht Patient Oriented Database (UPOD), an infrastructure of relational databases comprising data on patient characteristics, laboratory test results, medication orders, hospital discharge diagnoses and medical procedures for all patients treated at the University Medical Centre Utrecht (UMC Utrecht) was recently established. In this paper the structure, current content and potential applications of UPOD are presented. Because of the innovative character and clinical relevance of the linkage of laboratory and medication data, which increases the opportunities to study the use and effects of drugs in a clinical setting (i.e., clinical pharmacoepidemiological research), this specific feature is used as an example to illustrate the potential of UPOD. 
Table 1 Number of data within UPOD from the year 2005.

\begin{tabular}{lr}
\hline Type of data & Number \\
\hline Inpatient admissions & 28,561 \\
Day-care treatments & 15,305 \\
Outpatient visits & 333,858 \\
Laboratory test results & $3,812,756$ \\
Medication orders & 289,878 \\
Discharge diagnoses & 88,216 \\
Procedures & 148,499 \\
\hline
\end{tabular}

\section{Utrecht Patient Oriented Database: UPOD}

\section{Setting}

The UMC Utrecht is a 1042-bed academic medical centre located in the centre of The Netherlands. Approximately 165,000 patients are treated annually during more than 28,000 clinical hospitalisations, 15,000 day-care treatments and 333,000 outpatient visits (Table 1). At UMC Utrecht all administrative and clinical information on in- and outpatients is registered and processed electronically and stored at patient level within systems that are integrated in the hospital information system.

\section{Research database}

UPOD is a relational database infrastructure capturing complete and detailed data on patient characteristics, laboratory test results, medication orders, hospital discharge diagnoses and therapeutic procedures for all patients treated at UMC Utrecht since January 2004 (Figure 1). Periodically all relevant data are automatically transferred from the specific registration systems into tables in the management information system, which is an environment (SQL server) that allows checking, cleaning, storing, maintaining, questioning and linking of data (Figure 1). All data contain a patient identifier and an index date, allowing selection of unique patients or events and deterministic

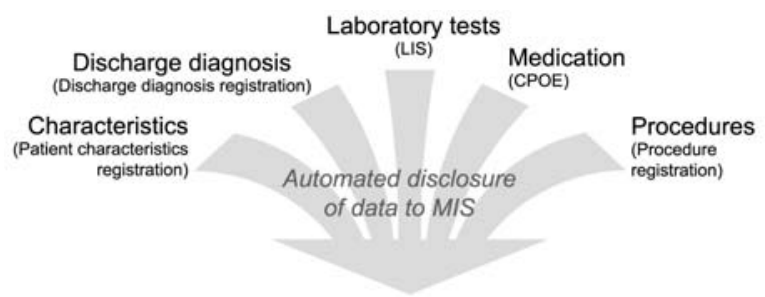

UPOD: control, cleaning, privacy constraints, linkage, storage

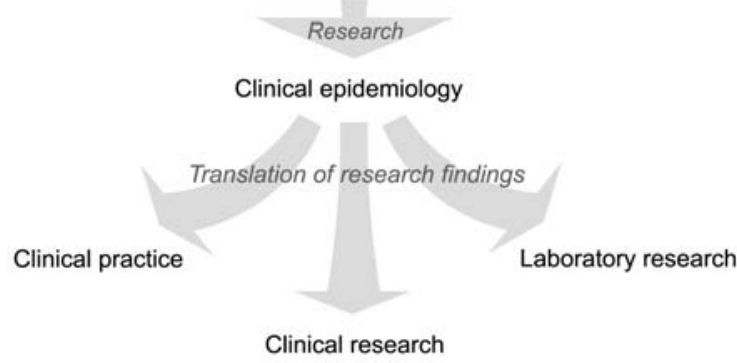

Figure 1 UPOD: clinical epidemiological research with a relational database system comprising patient-oriented clinical and administrative data. linkage between tables comprising different types of data. Researchers who are granted access to the data can make data selections in the management information system using SOL syntaxes. Subsequently, data can be downloaded from the management information system to the researcher's personal computer.

Data on patient characteristics are extracted from the hospital's central electronic patient registry and consist of gender and dates of birth, death, hospitalisation and discharge. Laboratory data originate from the laboratory information system, and include all tests concerning clinical chemistry, haematology, endocrinology, immunology and therapeutic drug monitoring. The records contain information on the collection date, type of material and the result. Medication data concern drugs that are ordered in the computerised physician order entry (CPOE) system for medication. Medication records contain information on the start and stop date, duration, prescriber (type of medical specialty), amount administered, dosage regimen, and route of administration for each drug prescription. Drugs are coded with regard to the different national (generic product code, trade code) (3) and international (Anatomical Therapeutic Chemical classification, ATC) (4) classification schemes. Diagnostic data concern the full list of discharge diagnoses (up to 10 diagnoses per admission) that are registered primarily for reimbursement purposes. Discharge diagnoses are coded according to the International Classification of Diseases, 9th edition Clinical Modification (ICD-9-CM) (5). Likewise, data on therapeutic procedures performed by medical specialists are registered. Procedures are coded according to the Classification of Procedures by Medical Specialists, published by the Dutch CBV Foundation (6).

In addition to the laboratory data described above, UPOD contains a specific database with haematology data on automated blood cell analyses performed with Abbott Cell-Dyn Sapphire automated blood cell analysers used at UMC Utrecht. A feature of this type of blood cell analyser is that it not only reports the parameters requested by the physician, but all haematological parameters that it is capable of measuring (7). For example, when a physician requests a haemoglobin measurement, the analyser also automatically determines the platelet count. Although this platelet count is not reported to the clinician, the analyser stores the data. Periodically, all data captured within the blood cell analysers are manually downloaded to a database format, and are cleaned and checked for integrity, making the data available for research. These haematological data include the collection date, type of material and the results, including flagging parameters.

\section{Ethical and privacy considerations}

The collection, storage and use of administrative and clinical patient information for scientific research is subject to ethical and privacy regulations $(8,9)$. The establishment and utilisation of UPOD is in accordance with guidance of the Institutional Review Board (IRB) and privacy board of UMC Utrecht, which allows 
the use of clinical data from patients who did not object to use of their data for scientific purposes, as long as the patients cannot be identified directly from the data.

Within UPOD, only data are captured that were initially registered during routine care and not for research purposes. Because no extra material, for example, blood samples, is taken from patients, there is not a requirement to obtain informed consent from individual patients or seek IRB approval for every study protocol.

At our institution, patients are informed at the time of admission that their data can be used for scientific research purposes. Patients can object to the use of their data within UPOD according to a general procedure for objecting to the use of data for scientific research that is available at UMC Utrecht.

To prohibit the identification of individual patients within the database, sensitive patient data must be encoded before they are processed outside the protective environment of the hospital and management information systems. For this purpose, the original patient identification number for UMC Utrecht is encrypted into a unique UPOD patient identifier within the database. Decrypting the patient identifier is possible in case it is essential to retrieve additional information from the patient's medical record. However, decryption is only possible after approval of the protocol by the IRB.

\section{Linking laboratory and medication data}

Laboratory data are often essential for selection, dosing and monitoring of drug therapy. Currently, many hospitals implement CPOE systems for ordering prescriptions or laboratory tests that contain decision support tools involving linkage of laboratory and pharmacy information at the time of ordering medication. This so-called real-time linkage of laboratory and medication information is considered an important contribution to reducing prescription errors and improving patient care (10) since, in the absence of such computerised support systems, patient safety hinges on the ability of the physician to recall a particular warning concerning a specific drug in relation to the clinical characteristics of the patient (11).

In addition to the benefits of real-time linkage for clinical practice, laboratory and medication data can also be linked retrospectively for research purposes within a database (10), as is done within UPOD. This innovative technique provides numerous opportunities to conduct pharmacoepidemiological studies in which the role of the clinical laboratory is considered. These include evaluating the quality of pharmacotherapy with regard to laboratory monitoring, studying therapeutic and adverse effects of drugs, and investigating drug-test interference (10).

In the following, examples of pharmacoepidemiological studies concerning the clinical laboratory are presented to illustrate the relevance of linking laboratory and medication data within a research database. We consider etiological (causality of an association between exposure and outcome), descriptive (pattern and frequency of the disease), prognostic (prediction of an outcome from factors that can be obtained before or at a certain time of treatment) and diagnostic (development of tests that allow accurate diagnosis of health status) epidemiological studies. Table 2 presents examples of pharmacoepidemiological studies currently being conducted within UPOD.

\section{Etiological epidemiology: adverse drug reactions and drug-test interference}

Adverse drug reactions Adverse drug reactions are considered a major threat to patient safety (17). Depending on the definition, adverse drug reactions occur in up to $5 \%-30 \%$ of hospitalised patients (18). Laboratory testing can be helpful in managing the risk of adverse drug reactions, as it has been shown that $60 \%-65 \%$ of clinically relevant adverse drug reactions can be detected with a biochemical test (13, 19-21). Several studies (22-25) have shown that linking laboratory and medication data for large groups of patients is a powerful tool for studying the association between adverse events that can be detected with a

Table 2 Examples of current research projects within UPOD.

\begin{tabular}{|c|c|c|}
\hline Subject & Type of epidemiology & \\
\hline $\begin{array}{l}\text { Laboratory monitoring for } \\
\text { heparin-induced } \\
\text { thrombocytopaenia (HIT) }\end{array}$ & Descriptive & $\begin{array}{l}\text { In patients at risk for HIT, close monitoring of the platelet } \\
\text { count and an anti-heparin platelet factor } 4 \text { antibody } \\
\text { test are advised to rule out HIT in case of suspicion (12); it } \\
\text { is investigated if there is a need to intensify laboratory } \\
\text { monitoring within our institution. }\end{array}$ \\
\hline $\begin{array}{l}\text { Epidemiology of drug-associated } \\
\text { blood dyscrasias }\end{array}$ & Etiologic & $\begin{array}{l}\text { Blood dyscrasias following exposure to non-cytotoxic } \\
\text { drugs are rare; however, the outcome can be severe, espe- } \\
\text { cially since they are often unexpected and are diagnosed } \\
\text { after symptoms occur; thrombocytopenia, agranulocytosis, } \\
\text { and aplastic anaemia are among the most reported and } \\
\text { fatal adverse drug reactions (13), but research into the fre- } \\
\text { quency, risk factors and mechanisms is still scarce }(14,15) \text {. }\end{array}$ \\
\hline $\begin{array}{l}\text { Laboratory markers for early } \\
\text { warning of drug-induced blood } \\
\text { dyscrasias }\end{array}$ & Prognostic & $\begin{array}{l}\text { Haematological parameters can possibly serve as early } \\
\text { warning markers of drug-induced blood dyscrasias; some } \\
\text { haematological parameters reflect blood dyscrasias in an } \\
\text { early stage and thus may be useful as indicators for } \\
\text { predicting drug toxicity (16). }\end{array}$ \\
\hline
\end{tabular}


biochemical test and drug exposure. Two recent examples are the assessment of the incidence of drug-induced liver injuries based on serum values for liver enzymes during hospitalisation (26) and the quantification of the association between hyponatraemia and the use of serotonergic antidepressants in elderly patients (27). In addition to evidence on the association between drug exposure and an adverse event, epidemiological studies can provide knowledge on risk factors for adverse drug reactions. An example of such a study is the recent identification of treatment-related risk factors for hospital-acquired hyponatraemia (28). Knowledge of risk factors is important for the identification of patients at high risk of adverse drug reactions (18) to initiate prophylactic treatment or close monitoring for the development of adverse drug reactions (29).

Drug-test interference With more than 40,000 drugs described that affect laboratory test results $(30,31)$, drug-test interference is a relevant issue in clinical chemistry (32). The interference can be due either to a biological effect, e.g., the increase in serum concentration of the thyroid hormone $\mathrm{FT}_{4}$ by valproic acid (33), or to analytical interference, e.g., interference by aminoglycoside in total protein determination in urine (34). Drug-test interference can lead to misinterpretation of laboratory data, potentially resulting in unnecessary medical services and costs. Gronroos et al. extensively evaluated the literature on drug-test interference and recommended the development of a database system comprising linked laboratory and medication data for appropriate investigation of drugtest interference (35).

\section{Descriptive epidemiology: quality of pharmacotherapy}

In selecting a drug, the patient's physical condition can be a contraindication. By linking laboratory and medication data, it can be investigated whether the drug is appropriately prescribed to the patient. Using this approach, Schiff et al. revealed that at their institutions a large proportion of patients received potassium supplementation while hyperkalaemic (36). By linking prescription claim data and hospital admission records, Juurlink et al. showed that publication of the results of the Randomised Aldactone Evaluation Study (RALES) was associated with an abrupt increase in the rate of prescriptions for spironolactone and in hyperkalaemia-associated morbidity and mortality in heart failure patients also treated with ACE inhibitors (37).

On the other hand, laboratory measurements can also reveal conditions that require treatment. Schiff et al. uncovered patients who were not treated with levothyroxine after abnormal levels of thyroid-stimulating hormone (TSH) were found (38).

Patient groups with altered drug metabolism, such as patients with renal insufficiency, often require dose adjustments of specific drugs. Epidemiological studies can be used to evaluate the adherence to dosing instructions with regard to renal insufficiency, as shown by Chertow et al., who reported that $70 \%$ of medication orders were written for an inappropriately high dose or frequency (39), increasing the risk of developing adverse drug reactions.

For a number of drugs, laboratory monitoring for drug toxicity, e.g., drug-induced liver damage, blood dyscrasias and nephrotoxicity is warranted (40). In several cases of adverse drug reactions that have led to withdrawal of drugs from the market, a lack of appropriate laboratory monitoring played an important role (41). Several recent studies considering laboratory monitoring during drug exposure in outpatients showed that essential monitoring was performed in only a minority of patients at risk of severe adverse drug reactions (42-44).

Laboratory monitoring can also be warranted for efficacy of drug therapy, for example, measuring cholesterol goal attainment in statin treatment. Goettsch et al. linked outpatient laboratory data to prescription histories from community pharmacies and found that the percentage of patients who achieved the cholesterol level recommended in guidelines was low in practice (45).

\section{Prognostic and diagnostic epidemiology: markers for drug effects}

Several adverse drug reactions develop unexpectedly and are diagnosed when symptoms occur, for example, drug-induced thrombocytopaenia is often detected after spontaneous/excessive bleeding occurs (14). For the patient (irreversible harm) and for society (increased medical costs), it is relevant to investigate whether the risk of such adverse drug reactions can be predicted before initiation of the medication and hence even guide the choice of medication, or if these adverse drug reactions can be diagnosed at an early stage (i.e., before clinical symptoms occur). Laboratory parameters could potentially serve as prognostic or diagnostic markers for adverse drug reactions (46), for example, the occurrence of the typical drop in platelet count associated with heparin-induced thrombocytopenia (HIT) (47). Epidemiological studies within databases linking laboratory and medication data can contribute to the identification of predictive or prognostic markers and/or the development of algorithms for adverse drug reactions.

\section{Discussion}

\section{Application of UPOD for clinical epidemiological research}

Transfer of automated laboratory data from the laboratory information system into a relational database infrastructure makes laboratory data available for clinical epidemiological research. Linking laboratory data to other clinical data provides numerous opportunities to study the biochemical characteristics of diseased populations, the effects of medical therapy that can be detected with laboratory tests and contribute to the development of predictive and diagnostic markers and/or algorithms for disease. 
The application of automated database systems comprising observational data on patient characteristics, diagnoses, disease and therapy is already an established and widely used approach in the study of the effects of drugs in clinical settings (48), in particular with regard to the detection, verification and quantification of adverse drug reactions (49). The linkage of laboratory and medication data is especially innovative for database systems comprising data on in-hospital patients, such as UPOD. With regard to the general population, laboratory data have recently become available within some of the automated database systems used in pharmacoepidemiological research, for example the insurance-based Kaiser Permanente database $(40,43)$ and the Dutch populationbased PHARMO Record Linkage System (45). However, until recently, most database systems used to study drug use in populations comprised drug histories from community pharmacies or hospitals linked to registrations of morbidity or hospital-discharge diagnoses (48), but lacked laboratory data, thereby limiting the possibilities for conducting studies on adverse drug reactions that can be detected biochemically, as illustrated in a recent study carried out by our group (50). It was found that the underlying disease overshadows many clinical conditions and that comorbidity is seldom registered in the case of severe illness. This could result in underestimation of the number of cases and potential bias when using hospital discharge diagnoses only in (pharmaco)epidemiological research. Because laboratory data allow more sensitive detection of the outcome and thereby increase the study power, the potential of investigations regarding, for example, the association between drug exposure and hyponatraemia would be increased if cases were sampled from laboratory data (50). Taking this into account and considering the elevated risk of adverse drug reactions in hospitalised patients, the importance of laboratory information in drug therapy and the continuing introduction of new drugs with innovative mechanisms of action, a research platform that allows the linkage of laboratory and medication data for hospitalised patients promises to be a valuable tool for clinical epidemiological studies aimed at investigating the (adverse) effects of drugs.

\section{Quality and data management}

The use of database systems such as UPOD for clinical epidemiological research has several advantages. In contrast to ad hoc data collection, database systems allow the study of complete and validated data on a patient level for a large population over a prolonged period of time with relatively easy access and at low cost (51). Furthermore, the collection of data using electronic registration systems and by automated transfer can be considered less prone to mistakes and less expensive compared to manual data collection. In addition, the real-life setting makes the population representative of patients actually being treated in clinical practice (51).

Although the potential advantages of a database comprising clinical data are numerous, potential threats to epidemiological research using observational data should be considered, for example, missing data and misclassification that are to a certain extent inherent to the use of retrospectively gathered data (52). To ensure maximum completeness and integrity, the data within UPOD are collected automatically and are extensively checked by data processing experts, administrative personnel and researchers. Furthermore, a data dictionary in which the database content is described in detail is available for researchers.

UPOD was established after the introduction of a CPOE system for ordering medication in our hospital and currently comprises complete data for a period of 2 years for one institution. This may possibly limit the study of rare outcomes in the short term and the extrapolation of findings to other hospitals. However, the population covered will increase rapidly over time (Table 1), and cooperation with other hospitals will extend the possibilities.

We believe that the institutional basis of the database has several advantages. The setting within a large academic hospital guarantees optimal synergy between clinical and both diagnostic and basic research laboratories. In this way, the translation of research findings to clinical practice and the experimental laboratory becomes relatively easy and efficient. For example, when novel associations between drug exposure and abnormal blood-cell parameters reflecting damage to blood cells are found in epidemiological research, mechanistic hypotheses can be further investigated within the experimental laboratory setting using blood cell-specific in vitro systems (53). In addition, the institutional basis makes it possible to validate data relatively easily or gather additional data, for example, by retrieving information that is currently not available within UPOD, such as radiology reports or electrocardiograms, from the original patient chart or by contacting the patient through his or her physician.

UPOD can be further expanded with data on extramural patient care, e.g., medication histories from community pharmacies or visits to general practitioners, and potentially other important types of clinical information such as pathology and genetic data. With regard to the latter, worldwide initiatives are currently undertaken to collect genetic data within population databases to study gene-disease relationships to characterise individual patients with regard to disease subtype based on their genetic profile (54). Adding genetic data to UPOD will provide interesting research possibilities such as pharmacogenetics, i.e., investigating the role of genetic variation in the patient's response to pharmacotherapy (55).

\section{Conclusions}

Facilitating the linkage of laboratory data collected during routine clinical care within a database system to other patient-oriented records broadens the opportunities for clinical pharmacoepidemiological research. Although recently established, UPOD prom- 
ises to be invaluable for this type of research and should be exploited fully.

\section{Acknowledgements}

UPOD is the result of the close collaboration between the Department of Pharmacoepidemiology and Pharmacotherapy of the Utrecht Institute for Pharmaceutical Sciences (UIPS) of Utrecht University and several departments within UMC Utrecht. We are indebted to all our colleagues involved with the UPOD initiative, especially to Leslie Beks, Jacq. Berk, Martien Boerefijn, Evert Jan van den Brink, Theo Canters, Ludi van Dun, Albertus ten Hertog, Kirana van Oosterhout, André Ringeling, Marcel Schinkel, Patrick Souverein, Kees Valk and Ton Wesseling for their support in establishing and maintaining the databases. The authors are grateful to Dr. Helena Chon, for reviewing an earlier version of this paper.

\section{References}

1. Porth AJ, Lubke B. History of computer-assisted data processing in the medical laboratory. Eur J Clin Chem Clin Biochem 1996;34:215-29.

2. Sackett D, Haynes RB, Tugwell P, Guyatt GH, editors. Clinical epidemiology: a basic science for clinical medicine, 2nd ed. Boston, MA: Little, Brown \& Co., 1991.

3. Z-Index. [About the G-standard]. http://www.zindex.nl/ (in Dutch). Accessed July 6, 2006.

4. WHO Collaborating Centre for Drug Statistics Methodology. About the ATC/DDD system. http://www. whocc.no/atcddd/atcsystem.html. Accessed July 6, 2006.

5. CDC National Center for Health Statistics. International Classification of Diseases, 9th Revision, Clinical Modification (ICD-9-CM). http://www.cdc.gov/nchs/about/otheract/icd9/abticd9.html. Accessed July 6, 2006.

6. CBV Foundation. [About the classification of medical specialist procedures (CMSV)]. http://www.cbv.nl (in Dutch). Accessed July 6, 2006.

7. Muller R, Mellors I, Johannessen B, Aarsand AK, Kiefer $\mathrm{P}$, Hardy J, et al. European multi-center evaluation of the Abbott Cell-Dyn Sapphire hematology analyzer. Lab Hematol 2006;12:15-31.

8. Ohno-Machado L, Silveira PS, Vinterbo S. Protecting patient privacy by quantifiable control of disclosures in disseminated databases. Int J Med Inform 2004;73: 599-606.

9. Hodge JG Jr, Gostin LO, Jacobson PD. Legal issues concerning electronic health information: privacy, quality, and liability. J Am Med Assoc 1999;282:1466-71.

10. Schiff GD, Klass D, Peterson J, Shah G, Bates DW. Linking laboratory and pharmacy: opportunities for reducing errors and improving care. Arch Intern Med 2003;163: 893-900.

11. Gurwitz JH. Serious adverse drug effects - seeing the trees through the forest [editorial]. N Engl J Med 2006; 354:1413-5.

12. Warkentin TE, Greinacher A. Heparin-induced thrombocytopenia: recognition, treatment, and prevention: the Seventh ACCP Conference on Antithrombotic and Thrombolytic Therapy. Chest 2004;126(Suppl):311S37S.

13. Kelly WN. Potential risks and prevention, part 1: fatal adverse drug events. Am J Health Syst Pharm 2001;58: 1317-24.

14. van den Bemt PM, Meyboom RH, Egberts AC. Druginduced immune thrombocytopenia. Drug Saf 2004;27: $1243-52$.
15. van Staa TP, Boulton F, Cooper C, Hagenbeek A, Inskip $H$, Leufkens HG. Neutropenia and agranulocytosis in England and Wales: incidence and risk factors. Am J Hematol 2003;72:248-54.

16. Wyrick-Glatzel J, Conway-Klaassen J. Clinical utility of the IRF: assessment of erythroid regeneration following parvo B19 infection. Clin Lab Sci 2002;15:208-12.

17. Lazarou J, Pomeranz BH, Corey PN. Incidence of adverse drug reactions in hospitalized patients: a meta-analysis of prospective studies. J Am Med Assoc 1998;279: $1200-5$.

18. van den Bemt PM, Egberts $A C$, de Jong-van den Berg LT, Brouwers JR. Drug-related problems in hospitalised patients. Drug Saf 2000;22:321-33.

19. Azaz-Livshits T, Levy M, Sadan B, Shalit M, Geisslinger G, Brune K. Computerized surveillance of adverse drug reactions in hospital: pilot study. Br J Clin Pharmacol 1998;45:309-14.

20. Tegeder I, Levy M, Muth-Selbach U, Oelkers R, Neumann F, Dormann H, et al. Retrospective analysis of the frequency and recognition of adverse drug reactions by means of automatically recorded laboratory signals. $\mathrm{Br}$ J Clin Pharmacol 1999;47:557-64.

21. Levy M, Azaz-Livshits T, Sadan B, Shalit M, Geisslinger G, Brune K. Computerized surveillance of adverse drug reactions in hospital: implementation. Eur J Clin Pharmacol 1999;54:887-92.

22. Classen DC, Pestotnik SL, Evans RS, Burke JP. Computerized surveillance of adverse drug events in hospital patients. J Am Med Assoc 1991;266:2847-51.

23. Dormann H, Muth-Selbach U, Krebs S, Criegee-Rieck M, Tegeder I, Schneider HT, et al. Incidence and costs of adverse drug reactions during hospitalisation: computerised monitoring versus stimulated spontaneous reporting. Drug Saf 2000;22:161-8.

24. Jha AK, Kuperman GJ, Teich JM, Leape L, Shea B, Rittenberg $E$, et al. Identifying adverse drug events: development of a computer-based monitor and comparison with chart review and stimulated voluntary report. J Am Med Inform Assoc 1998;5:305-14.

25. Honigman B, Lee J, Rothschild J, Light P, Pulling RM, Yu $T$, et al. Using computerized data to identify adverse drug events in outpatients. J Am Med Inform Assoc 2001;8:254-66.

26. Bagheri H, Michel F, Lapeyre-Mestre M, Lagier E, Cambus JP, Valdiguie $P$, et al. Detection and incidence of drug-induced liver injuries in hospital: a prospective analysis from laboratory signals. $\mathrm{Br} \mathrm{J}$ Clin Pharmacol 2000;50:479-84.

27. Movig KL, Leufkens HG, Lenderink AW, van den Akker VG, Hodiamont PP, Goldschmidt HM, et al. Association between antidepressant drug use and hyponatraemia: a case-control study. Br J Clin Pharmacol 2002;53:363-9.

28. Hoorn EJ, Lindemans J, Zietse R. Development of severe hyponatraemia in hospitalized patients: treatment-related risk factors and inadequate management. Nephrol Dial Transplant 2006;21:70-6.

29. van den Bemt PM, Egberts AC, Lenderink AW, Verzijl JM, Simons KA, van der Pol WS, et al. Risk factors for the development of adverse drug events in hospitalized patients. Pharm World Sci 2000;22:62-6.

30. Tryding N, Tufvesson C, Sonntag O. Drug effects in clinical chemistry, 7th ed. Stockholm: AB Realtryck, 1996.

31. Salway JG. Drug-test interactions handbook, 1st ed. London: Chapman and Hall Medical, 1990.

32. Kroll MH, Elin RJ. Interference with clinical laboratory analyses. Clin Chem 1994;40:1996-2005.

33. Kailajarvi M, Takala T, Gronroos P, Tryding N, Viikari J, Irjala $\mathrm{K}$, et al. Reminders of drug effects on laboratory test results. Clin Chem 2000;46:1395-400. 
34. Marshall T, Williams KM. Total protein determination in urine: aminoglycoside interference. Clin Chem 2003;49: 202-3.

35. Gronroos P, Irjala K, Heiskanen J, Torniainen K, Forsstrom JJ. Using computerized individual medication data to detect drug effects on clinical laboratory tests. Scand J Clin Lab Invest Suppl 1995;222:31-6.

36. Schiff GD, Aggarwal HC, Kumar S, McNutt RA. Prescribing potassium despite hyperkalemia: medication errors uncovered by linking laboratory and pharmacy information systems. Am J Med 2000;109:494-7.

37. Juurlink DN, Mamdani MM, Lee DS, Kopp A, Austin PC, Laupacis $A$, et al. Rates of hyperkalemia after publication of the Randomized Aldactone Evaluation Study. N Engl J Med 2004;351:543-51.

38. Schiff GD, Kim S, Krosnjar N, Wisniewski MF, Bult J, Fogelfeld $L$, et al. Missed hypothyroidism diagnosis uncovered by linking laboratory and pharmacy data. Arch Intern Med 2005;165:574-7.

39. Chertow GM, Lee J, Kuperman GJ, Burdick E, Horsky J, Seger DL, et al. Guided medication dosing for inpatients with renal insufficiency. J Am Med Assoc 2001;286: 2839-44.

40. Raebel MA, Lyons EE, Chester EA, Bodily MA, Kelleher $\mathrm{JA}$, Long $\mathrm{CL}$, et al. Improving laboratory monitoring at initiation of drug therapy in ambulatory care: a randomized trial. Arch Intern Med 2005;165:2395-401.

41. Meyboom R, Gribnau F, Hekster Y, de Koning G, Egberts A. Characteristics of topics in pharmacovigilance in The Netherlands. Clin Drug Invest 1996;12:207-19.

42. Simon SR, Andrade SE, Ellis JL, Nelson WW, Gurwitz $\mathrm{JH}$, Lafata JE, et al. Baseline laboratory monitoring of cardiovascular medications in elderly health maintenance organization enrollees. J Am Geriatr Soc 2005; 53:2165-9.

43. Raebel MA, McClure DL, Simon SR, Chan KA, Feldstein $\mathrm{AC}$, Gunter MJ, et al. Frequency of serum creatinine monitoring during allopurinol therapy in ambulatory patients. Ann Pharmacother 2006;40:386-91.

44. Derijks HJ, De Koning GH, Mantel-Teeuwisse AK, Egberts AC. Limited adherence to safety instructions in drug leaflets. Br J Clin Pharmacol 2002;54:678-9.
45. Goettsch WG, Yin DD, Alemao E, Klungel OH, Stalenhoef $A F$, Herings RM. Statins are less effective in common daily practice among patients with hypercholesterolemia: the REALITY-PHARMO study. Curr Med Res Opin 2004;20:1025-33.

46. Kane-Gill SL, Dasta JF, Schneider PJ, Cook CH. Monitoring abnormal laboratory values as antecedents to druginduced injury. J Trauma 2005;59:1457-62.

47. Warkentin TE, Roberts RS, Hirsh J, Kelton JG. An improved definition of immune heparin-induced thrombocytopenia in postoperative orthopedic patients. Arch Intern Med 2003;163:2518-24.

48. Strom BL, Carson JL. Use of automated databases for pharmacoepidemiology research. Epidemiol Rev 1990; 12:87-107.

49. Stricker BH, Psaty BM. Detection, verification, and quantification of adverse drug reactions. Br Med J 2004; 329:44-7.

50. Movig KL, Leufkens HG, Lenderink AW, Egberts AC. Validity of hospital discharge International Classification of Diseases (ICD) codes for identifying patients with hyponatremia. J Clin Epidemiol 2003;56:530-5.

51. Schneeweiss S, Avorn J. A review of uses of health care utilization databases for epidemiologic research on therapeutics. J Clin Epidemiol 2005;58:323-37.

52. Zwetsloot-Schonk JH, Snitker P, Vandenbroucke JP, Bakker AR. Using hospital information systems for clinical epidemiological research. Med Inform (Lond) 1989;14: 53-62.

53. Wijk R, van Wesel AC, Thomas AA, Rijksen G, van Solinge WW. Ex vivo analysis of aberrant splicing induced by two donor site mutations in PKLR of a patient with severe pyruvate kinase deficiency. $\mathrm{Br} \mathrm{J}$ Haematol 2004;125:253-63.

54. Potter JD. Epidemiology informing clinical practice: from bills of mortality to population laboratories. Nat Clin Pract Oncol 2005;2:625-34.

55. Maitland-van der Zee AH, de Boer A, Leufkens HG. The interface between pharmacoepidemiology and pharmacogenetics. Eur J Pharmacol 2000;410:121-30.

Received July 6, 2006, accepted September 29, 2006 